\title{
Correlation Between Clinical Findings of Temporomandibular Disorders and MRI Characteristics of Disc Displacement
}

\author{
Raman Kumar ${ }^{1, *}$, Shambulingappa Pallagatti ${ }^{2}$, Soheyl Sheikh ${ }^{2}$, Amit Mittal $^{3}$, Deepak Gupta ${ }^{2}$ and \\ Sonam Gupta ${ }^{1}$
}

${ }^{I}$ Department of Oral Medicine and Radiology, Guru Nanak Dev Dental College and Research Institute, Sunam, Punjab, India; ${ }^{2}$ Department of Oral Medicine and Radiology, M.M. College of Dental Sciences and Research, Mullana, Ambala, Haryana, India; ${ }^{3}$ Department of Radio-diagnosis and Imaging, M.M. Institute of Medical Sciences and Research, Mullana, Ambala, Haryana, India

\begin{abstract}
Objectives: Temporomandibular joint (TMJ) dysfunction is a common condition that is best evaluated with magnetic resonance (MR) imaging. The first step in MR imaging of the TMJ is to evaluate the articular disk, or meniscus, in terms of its morphologic features and its location relative to the condyle in both closed- and open-mouth positions. Disk location is of prime importance because the presence of a displaced disk is a critical sign of TMJ dysfunction. However, disk displacement is also frequently seen in asymptomatic volunteers. It is important for the maxillofacial radiologist to detect early MR imaging signs of dysfunction, thereby avoiding the evolution of this condition to its advanced and irreversible phase which is characterized by osteoarthritic changes such as condylar flattening or osteophytes. Further the MR imaging techniques will allow a better understanding of the sources of TMJ pain and of any discrepancy between imaging findings and patient symptoms. Henceforth, the aim of the study was to evaluate whether MRI findings of various degrees of disk displacement could be correlated with the presence or absence of clinical signs and symptoms of temporomandibular disorders in symptomatic and asymptomatic subjects. Materials and Methods: In this clinical study, 44 patients (88 TMJs) were examined clinically and divided into two groups. Group 1 consisted of 22 patients with clinical signs and symptoms of TMDs either unilaterally or bilaterally and considered as study group. Group 2 consisted of 22 patients with no signs and symptoms of TMDs and considered as control group. MRI was done for both the TMJs of each patient. Displacement of the posterior band of articular disc in relation to the condyle was quantified as anterior disc displacement with reduction (ADDR), anterior disc displacement without reduction (ADDWR), posterior disc displacement (PDD). Results: Disk displacement was found in 18 (81.8\%) patients of 22 symptomatic subjects in Group 1 on MRI and 4 (18.1\%) were diagnosed normal with no disc displacement. In Group 2, 2 (9.1\%) of 22 asymptomatic patients were diagnosed with disc displacement while $20(90.1 \%)$ were normal. Sensitivity and Specificity tests were applied in both the groups to correlate clinical findings of TMD and MRI characterstics of disc displacement and results showed Sensitivity of $90 \%$ and Specificity of $83.3 \%$. Conclusion: Disk displacement on MRI correlated well with presence or absence of clinical signs and symptoms of temporomandibular disorders with high Sensitivity and Specificity of $90 \%$ and $83.3 \%$ respectively.
\end{abstract}

Keywords: Disc displacement, magnetic resonance imaging, temporomandibular joint disorders.

\section{INTRODUCTION}

Temporomandibular joint (TMJ) is defined as the area where the mandible articulates with the temporal bone of the cranium. It is certainly one of the most complex joints in the body $[1,2]$.

It is a diarthrodial type of joint, located directly on either side of the head where the mandibular fossa of temporal bone and the mandibular condyle articulate $[1,3]$.

According to American Academy of Orofacial Pain (Jeffery P. Okeson), temporomandibular disorders (TMD) are defined as "A collective term embracing a number of clinical problems that involve the masticatory muscles, temporomandibular joint and associated structures or both". They are considered to be a subclassification of musculoskeletal disorders and typically run a recurrent and chronic

*Address correspondence to this author at the Department of Oral Medicine and Radiology, M.M. College of Dental Sciences and Research, Mullana, Ambala, Haryana, India; Pin 133203; Tel: +917206619752;

E-mail: ramankansal111@gmail.com course with a substantial fluctuation of TMD signs and symptoms over time $[1,4]$

It is a well established fact that temporomandibular joint dysfunction is quite common affecting upto $28 \%$ of population worldwide $[2,5]$. Further it is also documented that the prevalence of TMD differs between studies, probably because of variations in methodology and definitions of TMD. According to Gopal et al., the prevalence of signs and symptoms of TMD was found to be $52 \%$ which was less than the prevalence found by Modi P et al. (68.6\%) and Ryalat et al. $(55 \%)$ but more than prevalence found by Mutalu $\mathrm{N}$ et al. (17\%) [3, 6-8].

TMD patients may have various signs like clicking noise, disturbance in mandibular movements and symptoms like facial pain, and TMJ pain. TMD signs have been estimated to occur approximately twice as commonly as symptoms [1]. The prevalence of signs varies from $40 \%$ to $75 \%$, and symptoms from $5 \%$ to $33 \%$ [1]. In general population, symptoms are most commonly pronounced between the age of 17-30 years, while in TMD patients, symptoms are mostly pro- 
nounced between the age of $20-40$ years [4]. The prevalence of signs and symptoms has been reported to be four times higher in women than men in younger population $[1,2,4,9]$.

Temporomandibular joint disorders (TMDs) comprise a wide number of articular and/or muscular conditions in the orofacial region. The articular disc in the human TMJ is assumed to behave as a shock absorber and distributor of the joint loads. But the exact contribution of this disc to the mechanics of the TMJ is still poorly understood $[1,10]$. However the main articular alterations are Disc displacement and degenerative changes in both soft and hard tissues of the TMJ [11].

Furthermore, disc displacement which is also known as internal derangement, is one of the most frequent disorders of TMJ which has been considered as an underlying mechanism in pathogenesis of TMJ dysfunction [12]. It is defined as "disruption within the internal aspects of TMJ whereby the disk is displaced from its normal functional relationship with the mandibular condyle and articular portion of temporal bone" [12]. It is associated with clinical symptoms such as pain, joint sounds and abnormal jaw functions.

Disc displacement may be either anterior displacement with reduction, anterior displacement without reduction or posterior displacement [3]. In Disc Displacement with Reduction (DDR), the disc is anterior to the condyle in the closed mouth position and returns to its normal position when jaw is opened. On the contrary, in Disc Displacement without reduction (DDWR), the disc is anterior to the condyle in the closed mouth position and does not return to its normal position when the jaw is opened, while in Posterior Displacement (PD), the posterior band of the disc is in apparent contact with the bilaminar zone and its anterior band is at a 2 o'clock or 3 o'clock position [13].

Degenerative bony changes of TMJ are characterized by the osteophytes, erosion and sclerosis of the condyle, articular eminence and glenoid fossa either singly or in combination [11].

These alterations are considered to be the radiological signs of osteoarthritis and have been demonstrated in painful TMJ. Although some authors have reported that degenerative bony changes in the TMJ are more frequent with nonreducing type of disc displacement, but the relationship between these conditions is not fully clear. However, it is hypothesized that bone changes or osteoarthritis is the progression of disc displacement, particularly in non reducing type of disc displacement [11].

Apart of the fact that the disc displacement is classified under TMDs, but the recent literature advocates that the disc displacement is prevalent in a substantial number of asymptomatic subjects. Rajane F. Ribeiro et al. in 1997 reported $25 \%$ cases of disc displacement on MRI evaluation in 56 asymptomatic volunteers [13]. Tore A. Larheim et al. in 2001 reported a $35 \%$ prevalence of internal derangement on MRI evaluation of 62 asymptomatic people [14].

MRI Examination of TMJ plays an important role in the diagnosis of internal derangement because it allows the direct visualization of articular disc in both open and close mouth position [7]. It can provide information on the position, morphology, signal intensity and structure of the disc.
Further it can also elaborate the quantity of synovial fluid, the bone marrow, peri-articular tissues, condition of the bone, posterior attachment and the retrodiscal tissues $[12,13$, $15]$.

Furthermore, MRI has been reported to be $95 \%$ accurate in assesement of disc position and form and $93 \%$ accurate in assesement of osseous changes $[2,11]$. Henceforth it was advocated that combined imaging in the sagittal and coronal planes provides good definition of the spatial relationship between the disc and the condyle and other structures of Temporomandibular joint $[12,13,16]$.

These MRI characteristics can relate to the pain and dysfunction of patients with TMD and thus are very important considerations in the treatment planning of TMD $[12,13]$.

Henceforth, the aim of the study was to evaluate the correlation between the presence and absence of signs and symptoms of temporomandibular joint disorders in patients and MRI findings of disc position.

\section{MATERIALS AND METHODOLOGY}

In this clinical study, 44 patients ( $88 \mathrm{TMJ}$ ) of either sex, between 20-40 years of age, reporting to the Department of Oral Medicine and Radiology were selected. All the subjects were divided into 2 groups. Group I consisted of 22 subjects with clinical signs and symptoms of temporomandibular disorders considered as Study group. Group II consisted of 22 subjects with no clinical signs and symptoms of temporomandibular disorders considered as Control group. Selection of subjects in Group I was based on the Research diagnostic criteria Axis 1-2011 by Daniele Manfredini, Inclusion and Exclusion criteria [17] as follows:

1. Clicking in the TMJ on both vertical range of motion either opening or closing, either unilaterally or bilaterally, which can be reproducible on two of three consecutive trials and is eliminated on protrusive opening.

2. Reduced mouth opening less than $35 \mathrm{~mm}$ along with deviation towards the ipsilateral side.

3. Pain as a complaint in the jaws, face, temple or inside the ear along with tenderness on palpation in at least three or more muscle sites which are located in only the masseter, temporalis and lateral pterygoid muscles, with or without reduced mouth opening.

4. Pain in one or both the joints sites on palpation along with pain as a complaint during maximum mouth opening and lateral excursion.

Clinical Examination: Each subject was seated on a dental chair with his or her head leaned slightly backwards on the headrest. The data such as name, age, sex, address, etc. were recorded in the proforma. A diluted $0.2 \%$ chlorhexidine gluconate mouthwash was given to rinse the oral cavity. The clinical examination was carried out wearing sterile gloves and mouthmask under artificial illumination. Patients were evaluated about the chief complaint and selected on the basis of four putative signs and symptoms of TMDs selected by RDC/TMD Axis 1-2011 by Daniele Manfredini and findings were recorded in the Proforma. 
To determine the joint sounds, the diaphragm of a stethoscope was placed over the skin surface over TMJ area and patients were asked to open and close the mouth three times. If joints sounds were present it was recorded in the Proforma.

To assess the maximum mouth opening, patients were asked to open the mouth three times as wide as possible and the greatest opening was recorded. The amount of maximum mouth opening was measured with a divider positioned in between the incisal edges of the maxillary and mandibular central incisors and recorded using a ruler.

Deviation on opening was visually observed by asking the individuals to close the mouth in centric occlusion and then to open slowly wide. The motion was repeated several times to watch for any deviation visually.

Tenderness was evaluated by palpating the muscles of mastication i.e. Masseter, Temporalis, Lateral Pterygoid, Medial Pterygoid and Sternocleidomastoid muscles bilaterally and both the joint sites pre-auricularly and intraauricularly in open and closed mouth position.

MRI Examination: The MRI investigation was carried out with the help of Digital MRI Scanner (1.5T, PHILIPS ACHIEVA, NETHERLANDS) with double surface coil 20 $\mathrm{cm}$ in diameter. The MRI procedure was explained verbally to the subjects and they were transferred to the Department of Radio diagnosis and Imaging. Each individual included in the study was subjected to the standard Temporomandibular joint open and close mouth imaging in sagittal plane using MRI with adequate protective measures. All the scans were performed on the same MRI scanner using the same protocols and the images were submitted to the digital manipulation in the workstation and recorded in Digital Imaging and Communication in Medicine (DICOM) format. All the images in the DICOM format were taken in the empty compact disc and prints out of the selected images were obtained. Tracing paper was placed over each selected printout. The condyle, glenoid fossa, articular eminence, articular disc and other associated structures were traced. The relationship between position of articular disc and the condyle was carried out.

The position of the disc was classified as follows:

1. Normal State $(N)$ : The posterior band of the disc is centered in relation to the condyle and the bottom of the glenoid fossa.in closed mouth position (Fig. 1) and in open mouth position head of the condyle articulates central zone of articular disc (Fig. 2)

2. Disc Displacement with Reduction (DDR): The disc is anterior to the condyle in the closed mouth position and returns to its normal position when the jaw is opened (Fig. 3, 4).

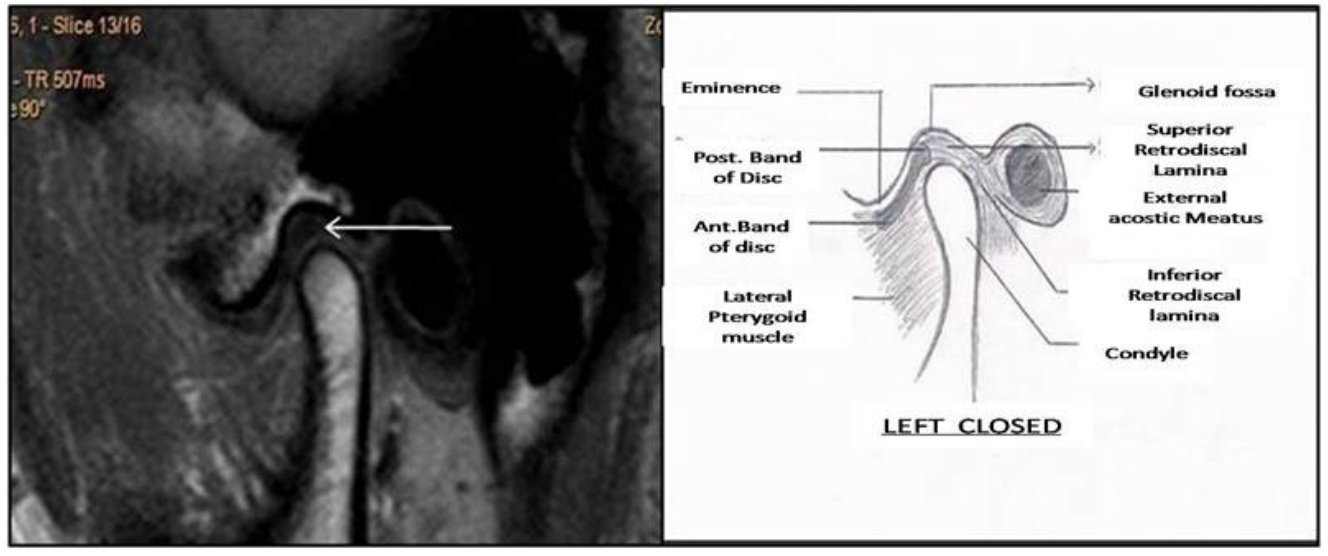

Fig. (1). MR image showing normal disc-condyle relationship in closed mouth position.

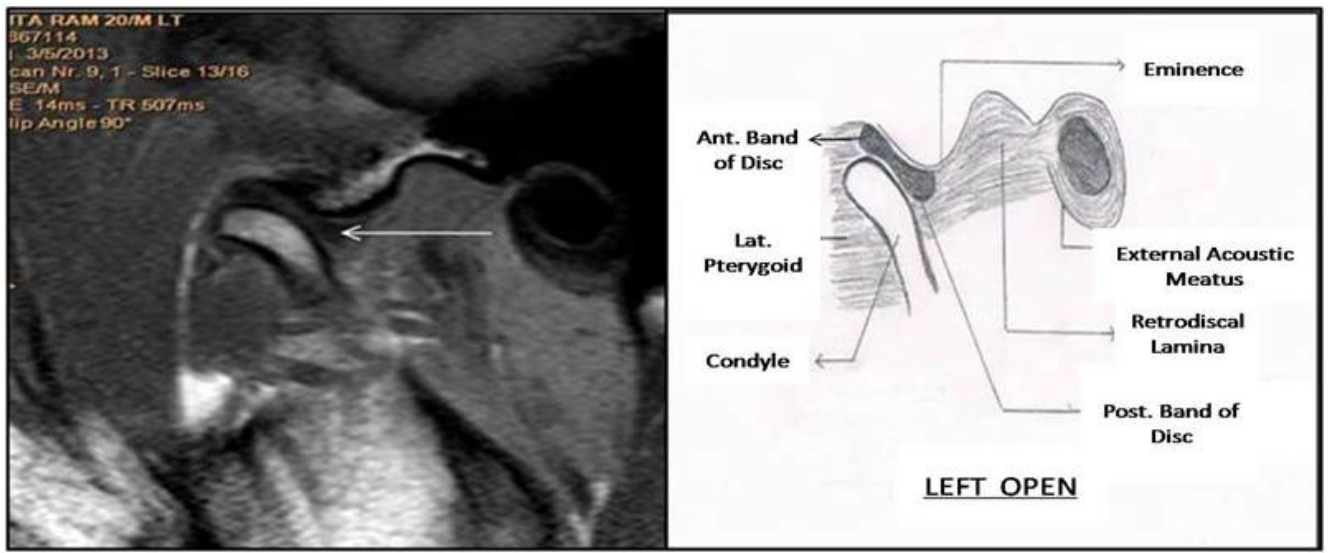

Fig. (2). MR image showing normal disc-condyle relationship in open mouth position. 


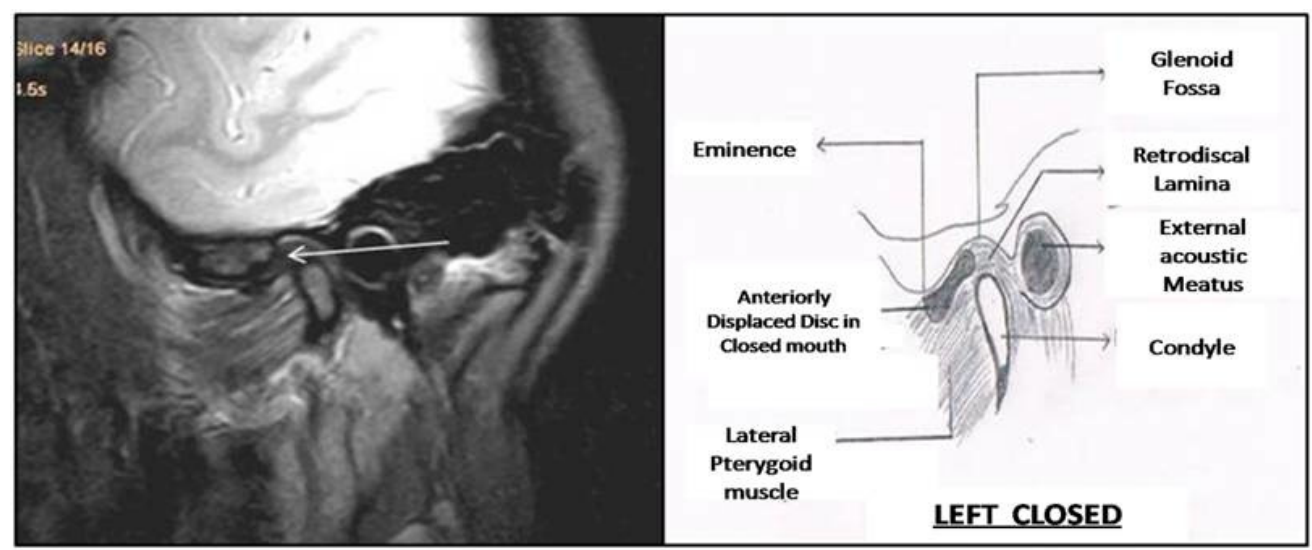

Fig. (3). MR image showing Anterior Disc Displacement in closed mouth position.

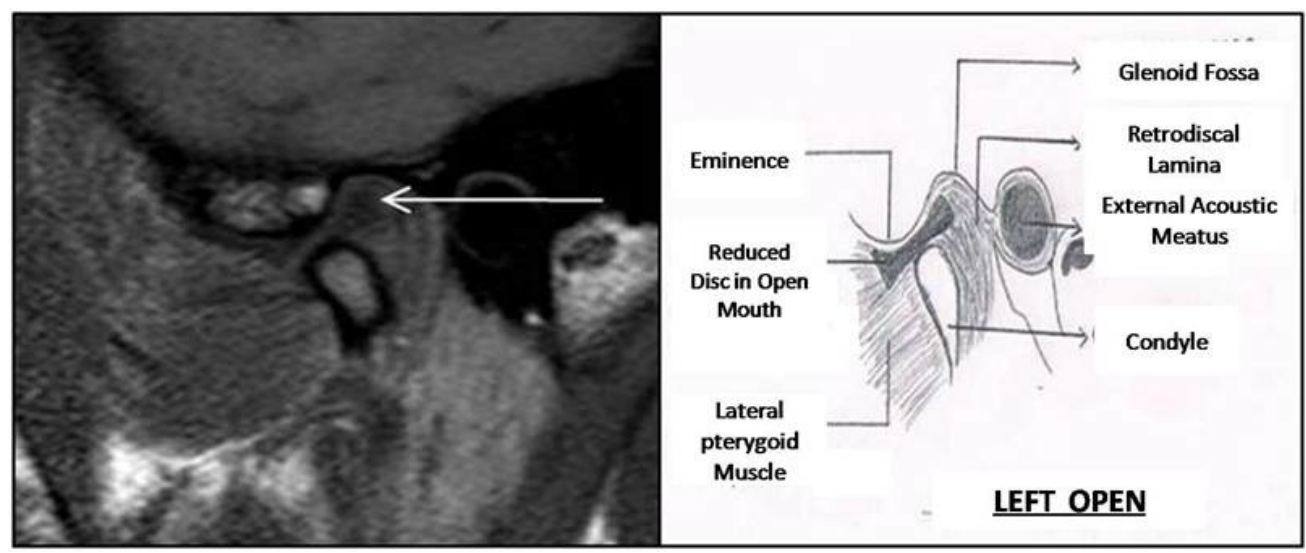

Fig. (4). MR image showing Disc Reduction in open mouth position.

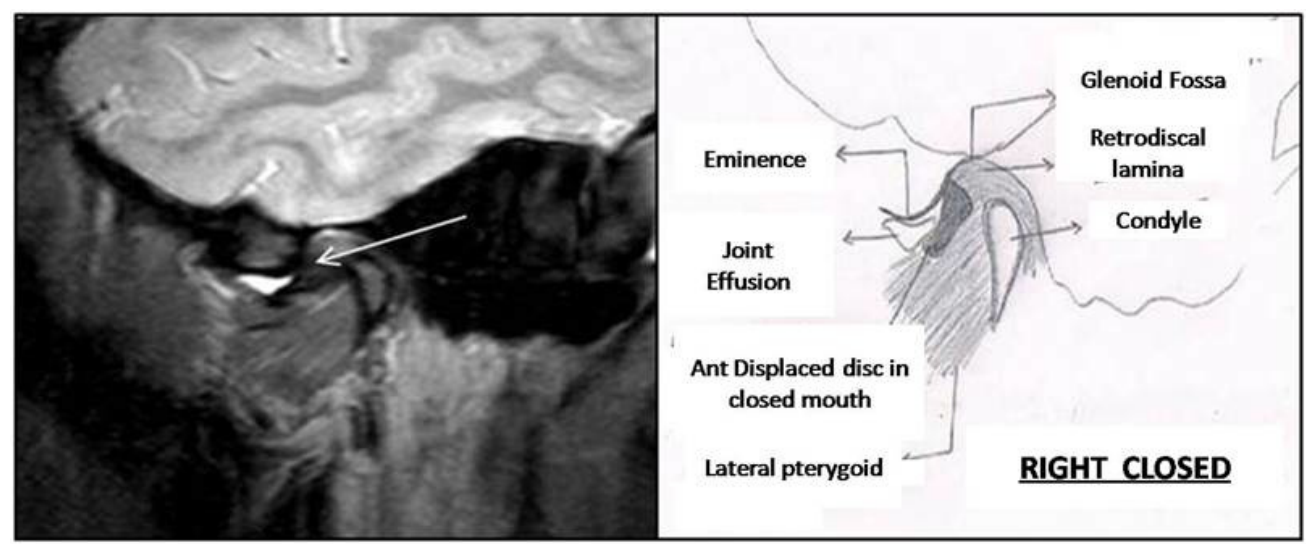

Fig. (5). MR image showing Anterior Disc Displacement in closed mouth position.

3. Disc Displacement without Reduction (DDWR): The disc is anterior to the condyle in the closed mouth position and does not return to its normal position when the jaw is opened (Fig. 5, 6).

4. Posterior Displacement (PD): The posterior band of the disc is in apparent contact with the bilaminar zone and its anterior band is at a 2 o'clock or 3 o'clock position.

The obtained data were subjected to statistical analysis for evaluation.

The statistical analysis was carried using Stastical Package for Social Sciences (SPSS Inc., Chicago, IL, Version
15.0 for windows). All the quantitative variables were estimated using measure of central location (Mean, Median) and measures of dispersion (Standard Deviation). Proportions were compared using Chi square test. Sensitivity and Specificity (Vassar Stats) were applied for correlating clinical findings and MRI characterstics.

\section{RESULTS AND ANALYSIS}

In both the groups, all the subjects were in the range of 20-40 years. In Group 1, out of 22 symptomatic subjects, 13 $(59.1 \%)$ subjects were male and $9(40.9 \%)$ were female. The minimum age in Group 1 was 20 years and maximum age 


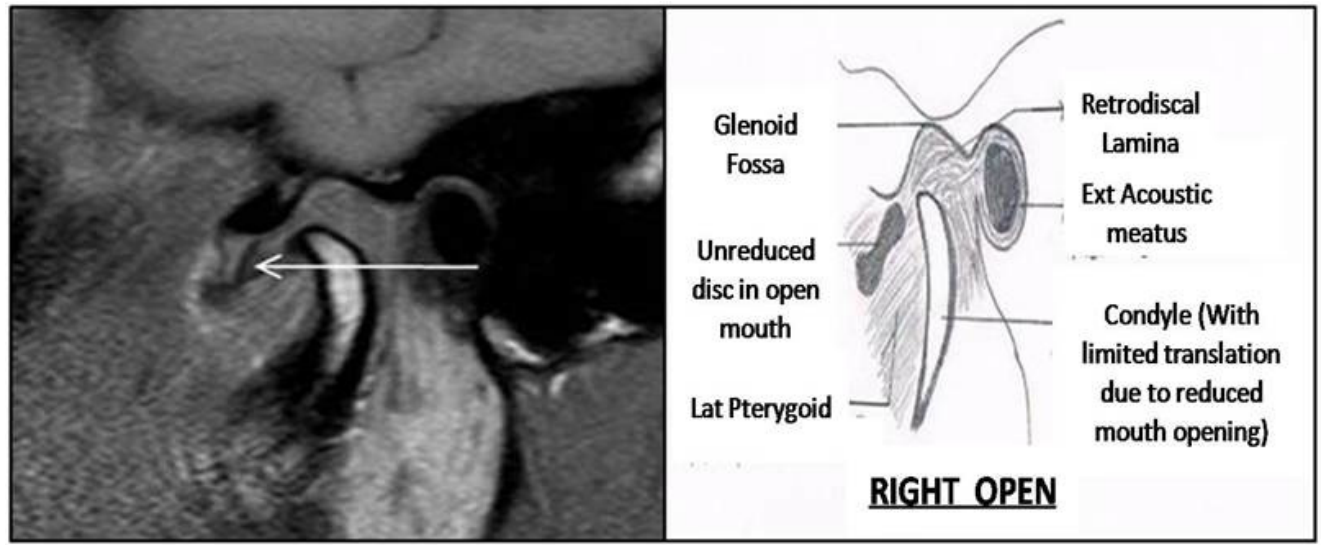

Fig. (6). MR image showing Non-Reduced Disc in open mouth position.

Table 1. Distribution of patients in Group 1 on the basis of clinical diagnosis.

\begin{tabular}{|c|c|c|}
\hline Clinical Diagnosis & No. of Patients & Percentage \\
\hline \hline LT and RT TMD & 4 & 18.2 \\
\hline LT sided TMD & 12 & 54.5 \\
\hline RT sided TMD & 6 & 27.3 \\
\hline Total & 22 & 100.0 \\
\hline
\end{tabular}

Table 2. Presence of articular disc displacement in Group 1 and Group 2 on MRI.

\begin{tabular}{|c|c|c|c|}
\hline Groups & $\begin{array}{c}\text { Total } \\
\text { N }\end{array}$ & Clinically Diagnosed TMD & DD Diagnosed by MRI \\
\hline \hline $\begin{array}{c}\text { Group 1 } \\
\text { (Symptomatic) }\end{array}$ & 22 & 22 & $18(81.8 \%)$ \\
\hline $\begin{array}{c}\text { Group 2 } \\
\text { (Asymptomatic) }\end{array}$ & 22 & 0 & $2(9.1 \%)$ \\
\hline
\end{tabular}

was 40 years. The mean age in Group 1 was $26.55 \pm 6.82$. In Group 2, out of 22 asymptomatic subjects, 10 (45.65) subjects were male and $12(54.4 \%)$ were female. The minimum age in Group 2 was 23 years and maximum age was 32 years. The mean age in Group 2 was $25.90 \pm 2.10$.

Out of total 22 patients in Group 1 (Table 1), 4 (18.2\%) were clinically diagnosed as Bilateral TMD, $12(54.5 \%)$ were clinically diagnosed as Left TMD and 6 (27.3\%) were clinically diagnosed as Right TMD.

In Group 1, out of 22 symptomatic subjects, 18 (81.8\%) subjects were diagnosed with Disc Displacement by MRI. In Group 2, out of 22 asymptomatic subjects, 2 (9.1\%) subjects were diagnosed with Disc Displacement by MRI. The presence of articular disc displacement by MRI showed the highly significant difference in between Group 1 and Group 2 with $\mathrm{P}=0.00$ (Table 2).

Out of total 22 patients in Group 1 (Table 3), 4 (18.2\%) were diagnosed as Normal. Left and Right sided Anerior Disc Displacement with reduction was found in $2(9.15)$ and $4(18.2 \%)$ patients respectively. $5(22.7 \%)$ patients were diagnosed as left sided Anterior Disc Displacement without
Reduction. Only 1(4.5\%) patient was diagnosed as Right sided Anterior Disc Displacement without Reduction, while 1 (4.5\%) patient presented with Left sided Posterior Disc Displacement. $2(9.1 \%)$ patients were diagnosed as Bilateral TMD with Left sided Anterior Disc Displacement without Reduction and Right sided Anterior Disc Displacement with Reduction. 3 (13.6\%) were diagnosed as Bilateral TMD with Left sided Anterior Disc Displacement with Reduction and Right sided Anterior Disc Displacement without Reduction.

Out of total 22 asymptomatic subjects in Group 2, 20 (90.95) subjects were diagnosed as normal with no disc displacement on MRI, while $2(9.1 \%)$ subjects were diagnosed with only Right sided Anterior disc Displacement with Reduction.

Table 4 depicts the MRI findings of TMJs evaluated in Group 1 (Symptomatic subjects) and Group 2 (Asymptomatic subjects). In Group 1, out of $44 \mathrm{TM}$ joints, based on MRI findings, $21(47.7 \%)$ joints were diagnosed as normal with no disc displacement, $11(25.0 \%)$ were diagnosed as Anterior Disc Displacement with Reduction, 11(25.0\%) were diagnosed as Anterior Disc Displacement without Reduction 
Table 3. Distribution of patients in Group 1 on the basis of type of disc displacement based on MRI findings.

\begin{tabular}{|c|c|c|}
\hline MRI Diagnosis & No of Patients & Percentage \\
\hline \hline NORMAL & 4 & 9.2 \\
\hline LT Ant. DDR & 2 & 18.2 \\
\hline RT Ant. DDR & 4 & 22.7 \\
\hline LT Ant. DDWR & 5 & 4.5 \\
\hline RT Ant. DDWR & 1 & 4.5 \\
\hline LT Post. DD & 1 & 9.1 \\
\hline LT Ant. DDWR, RT Ant. DDR & 2 & 13.6 \\
\hline LT Ant. DDR, RT Ant. DDWR & 3 & 100.0 \\
\hline Total & 22 & \\
\hline
\end{tabular}

Table 4. Evaluation of total disc displacements by MRI diagnosis between Group 1 and Group 2.

\begin{tabular}{|c|c|c|c|c|}
\hline MRI findings & Group 1 & Group 2 & Chi-square & Df value \\
\hline \hline Total no. of patients & 22 & 22 & & \\
\hline Total no of joints examined & 44 & 44 & - & - \\
\hline No DD & $21(47.7 \%)$ & $42(95.5 \%)$ & - & - \\
\hline Ant. DDR & $11(25 \%)$ & $2(4.5 \%)$ & - & - \\
\hline Ant. DDWR & $11(25 \%)$ & 0 & - & - \\
\hline Post. DD & $1(2.3 \%)$ & 0 & - & - \\
\hline Total disc displacement & $23(52.3 \%)$ & $2(4.5 \%)$ & 17.6 & - \\
\hline
\end{tabular}

while only $1(2.3 \%)$ was diagnosed as Posterior Disc Displacement. In Group, 2 out of 44 TM joints, $42(95.5 \%)$ joints were diagnosed as normal and $2(4.5 \%)$ joints were diagnosed as Anterior Disc Displacement with Reduction. Chi - square test revealed that the occurrence of total disc displacements between Group 1 and Group 2 was highly significant with $\mathrm{P}=0.00$.

In Group 1, out of 22 subjects clinically diagnosed as TMD on the basis of RDC/RMD, 18 (81.8\%) subjects were diagnosed with Disc Displacement on MRI and 4 (18.1\%) were diagnosed normal with no Disc Displacement on MRI. In Group 2, out of 22 asymptomatic subjects, 2 (9.1\%) were diagnosed with Disc Displacement, while 20 (90.1\%) were normal with no Disc Displacement on MRI. In both the groups, MRI findings of Disc Displacement correlated well with clinical findings of TMD with Sensitivity of $90 \%$ and specificity of $83.3 \%$ (Table 5).

In Group 1 with 22 symptomatic subjects, 44 TM joints were examined in which $26(59.09 \%)$ were symptomatic joints and $18(40.91 \%)$ were asymptomatic joints on the basis of clinical findings. Out of total 26 symptomatic joints diagnosed clinically, 16 joints were diagnosed Left sided TMD and 10 joints were diagnosed Right sided TMD clinically. But on MRI study, out of these 16 joints, 11 were diagnosed with disc Displacement of the same side and 5 joints with no Disc Displacement. Whereas out of 10 Right sided TMD, on MRI 8 joints were diagnosed with disc displacement, while 2 joints with no disc displacement. Thus in total, $19(73.1 \%)$ joints depicted disc displacement on MRI and 7 (26.9\%) depicted no disc displacement. Further, out of 18 asymptomatic joints, $4(22.2 \%)$ joints were diagnosed with disc displacement on MRI and $14(77.8 \%)$ were diagnosed with no disc displacement. Out of 4 joints, 3 (16.7\%) were diagnosed as Ant. Disc Displacement with Reduction and 1 (5.6\%) was diagnosed as Ant. Disc Displacement without Reduction. In Group 2, 44 asymptomatic TM joints were examined by MRI. Only 2 (4.5\%) joints showed Disc Displacement on MRI.

Sensitivity and specificity tests (Vassar stats) were applied. It implied the $82.6 \%$ sensitivity and $66.7 \%$ specificity in Group 1 between MRI diagnosis and clinical findings. Whereas in Group 2, sensitivity was $0 \%$ because no clinically symptomatic joint was there and specificity was 100\% (Table 6).

\section{DISCUSSION}

Disc displacement which is also known as internal derangement, is one of the most frequent cause of TMJ disorders which has been considered as an underlying mechanism in pathogenesis of TMJ dysfunction [5]. 
Table 5. Correlation between presence of Disc Displacement on MRI with clinical findings of TMD in patients of Group 1 and Group 2.

\begin{tabular}{|c|c|c|c|c|c|c|}
\hline Groups & \multicolumn{2}{|c|}{ TMD based on clinical findings } & \multicolumn{2}{|c|}{ Disc Displacement on MRI findings } & $\begin{array}{l}\text { Sensitivity } \\
\text { (Sn.) }\end{array}$ & $\begin{array}{c}\text { Specificity } \\
\text { (Sp.) }\end{array}$ \\
\hline Group 2 (22 subjects) & $0(0.0)$ & 22 & $2(9.1)$ & $20(90.1)$ & $90 \%$ & $83.3 \%$ \\
\hline
\end{tabular}

Table 6. Correlation between MRI findings of Disc Displacement and clinical diagnosis of TMD in the joints of Group 1 and Group 2.

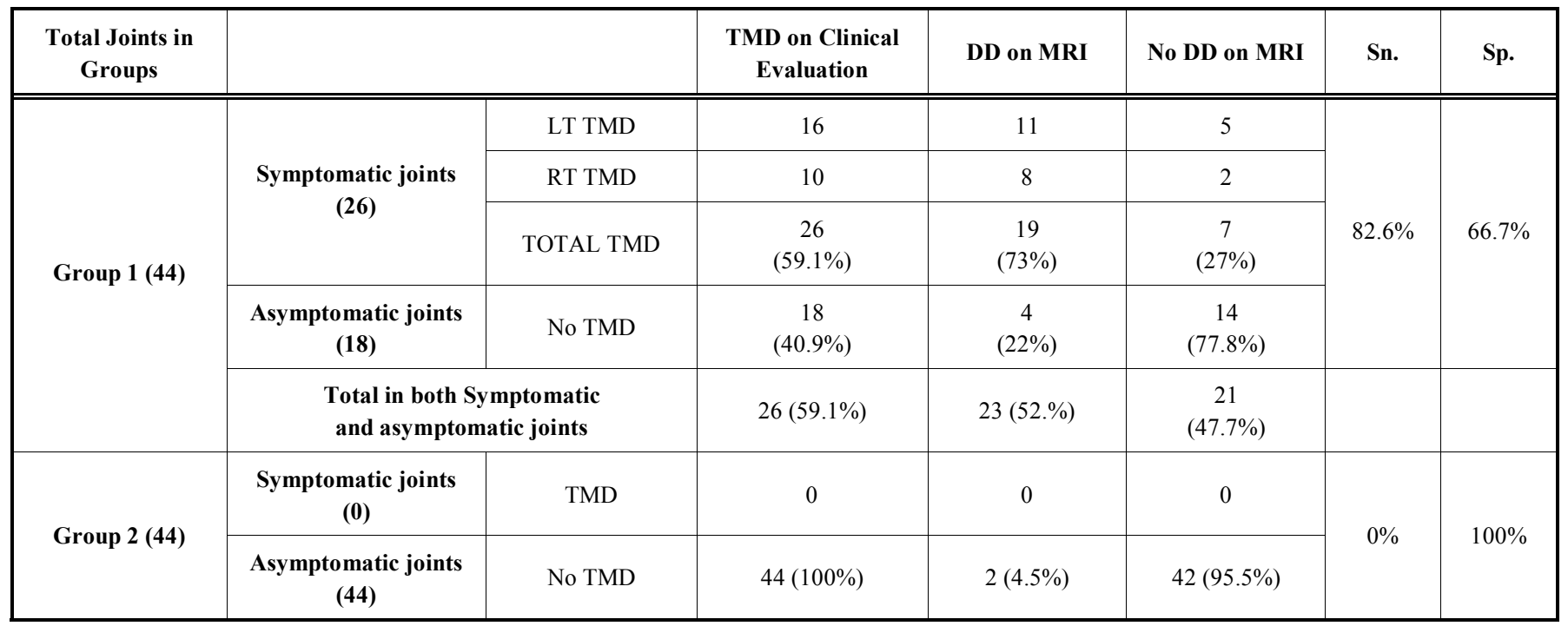

Further, various authors have debated regarding the role of presence of Disc Displacement on MRI and presence of signs and symptoms of TMJ disorders [10]. Apart of the fact that this aspect is a topic of debate, no concrete conclusions have been achieved. Henceforth, the present study was focused on evaluating the presence of signs and symptoms of TMDs in symptomatic and asymptomatic subjects and correlating these findings with MRI findings of Disc Displacement.

Various authors have proposed different criteria for the diagnosis, treatment planning and for the research purpose for defining subjects groups. These criteria include Research diagnostic criteria, American academy of Craniomandibular disorders guidelines (1990), Guidelines of the American Academy of Orofacial Pain (2008) and Craniomandibular Index Criteria (CMI) by Friction and Schiffman (1986) [18, 19]. But in this study, Research Diagnostic Criteria Axis 1 2011 was followed because it is latest and well accepted for TMDs [20].

Various authors have commented on the use of different radiographic approaches for the TMJ examination which includes Panoramic radiography, Computed Tomography and MRI. MRI Examination of TMJ has gained an important role in diagnosis of disc displacement because it allows the direct visualization of articular disc in both open and close mouth position [5].

It was found that $81.8 \%$ of symptomatic subjects presented with Disc Displacement on MRI examination. These results were in agreement with previous studies done by Kannan A et al, Ribeiro RF et al. and Milano V et al. who reported the prevalence of disc displacement in the range of $77 \pm 91 \%[2,10,21]$.

On the contrary, recent literature also advocates the presence of disc displacement in a number of asymptomatic subjects. In this study, $9.1 \%$ of the asymptomatic subjects presented with Disc Displacement which was in agreement with various studies in literature $[10,14]$ In addition, many authors reported higher prevalence of Disc Displacement in asymptomatic subjects like Ribeiro RF et al. in 1997 reported $25 \%$ cases of disc displacement while, Larheim TA et al. in 2001 reported a 35\% prevalence of Disc Displacement on MRI evaluation in asymptomatic subjects [10, 14].

When comparison was done between Group 1 and Group 2 for the presence of Disc Displacement based on MRI findings, there was highly significant difference in the presence of Disc Displacement with $P$ value of 0.00 .This result was in agreement with Larheim et al. [14, 22, 23].

According to various authors, although disc displacement is undoubtedly very common in symptomatic patients, its significance for the presence in asymptomatic subjects is difficult to interpret [21]. In the 1970s, it was suggested that after disc displacement, pain and dysunction was caused by the condyle pressing on the highly vascularised, innervated bilaminar zone or alternatively the pain might be due to pressure on, and/or distension of the joint capsule by the dis- 
placed disc [27] All these findings suggest that disc displacement alone is unlikely to be the primary cause of pain and dysfunction, but disc displacement is now held to be a predisposing factor that increases the risk of signs and symptoms of TMD [21, 24, 25].

In this present study, $25 \%$ of the joints showed Anterior Disc Displacement with reduction and the same number i.e. 25\% showed Anterior Disc Displacement without reduction on MRI in Group 1 (Symptomatic subjects). Posterior Disc Displacement was found to be very rare i.e. $2.3 \%$. This may be attributed to the fact that the normal position of the disc is anterior to the condyle [27]. However, in Group 2, Disc Displacement was found in 2 joints and both were diagnosed with Disc Displacement with reduction. Further, in 18 asymptomatic joints of Group 1, 4 joints showed Disc Displacement in which 3 joints were with Disc Displacement with reduction and 1 joint showed Disc Displacement without reduction. These results were in agreement with previous studies done by Maizlin ZV et al., Kannan et al. and Sener S et al. Maizlin recorded 37\% joints with DDR and $17 \%$ with DDWR in symptomatic joints, whereas in asymptomatic joints, 13 joints showed Disc Displacement and all were with DDR [11]. However, Sener reported 65\% joints with DDR and $35 \%$ with DDWR. Furthermore, Kannan et al. recorded $63 \%$ joints with DDR and $10 \%$ joints with DDWR. From this we can conclude that there is higher prevalence of Disc Displacement with reduction in symptomatic and asymptomatic joints $[2,12,13]$.

Further in this present study, MRI findings of Disc Displacement correlated well with clinical findings of TMD in patients of Group 1 and Group 2 with Sensitivity of $90.0 \%$ and specificity of $83.3 \%$. Considering joints in Group 1, Sensitivity and Specificity was $82.6 \%$ and $66.7 \%$ respectively. In Group 2, Sensitivity was $0 \%$ because all the joints were asymptomatic, while Specificity was $100 \%$. Related studies have been done by various authors. They recorded variations in sensitivity and specificity of MRI for Disc Displacement comparing with clinical findings i.e. Benbelaid $\mathrm{R}$ et al. (Sensitivity of $63 \%$, Specificity of $81 \%$ ), Shaefer JR et al. (Sensitivity 94\%, Specificity of 36\%) and Aoyama S et al. (Sensitivity of $95.65 \%$, Specificity 71.43\%) [26-28]. The reason for the variations may be attributed to the fact that they have taken the different criteria for diagnosing TMD and different protocols for diagnosing Disc Displacement on MRI.

Keeping these results in mind, it can be tentatively concluded that MRI is highly sensitive and specific for the Disc Displacement and the diagnosis made with MRI can be correlated well with clinical signs and symptoms of Temporomandibular joint disorders in symptomatic and asymptomatic subjects.

Further, it can also be commented that the above drawn conclusion would have been more concrete if the limitation of this study were dealt pertinently. In this study, sample size was small. Further, the Group 1 patients were selected according to RDC, but no concern was paid to the duration of the presence of these signs and symptoms. Furthermore, in this study, MR images were interpreted by a single Radiologist. Moreover, this study also does not correlate whether any particular clinical sign or symptom is related with the presence of Disc Displacement.

Henceforth, further studies are required to overcome the limitations of the present study and in substaining the above results.

\section{CONCLUSION}

Disc Displacement as observed with MRI correlated well with clinical signs and symptoms in cases of displacement of posterior band in relation to mandibular condyle and in cases without reduction when the mouth was opened. Also there is highly significant difference for Disc Displacement between symptomatic and asymptomatic subjects.

Under non-physiologic loading, the various structures of the TMJ show adaptative and compensatory responses to prevent permanent tissue damages and allow for function. Decompensation and degenerative changes may occur when TMJ modeling is unable to restore a biomechanical equilibrium. Disc derangements may lead to altered loading and regressive changes in the TMJ. However, because of the adaptative capacity of the joint, these changes may be asymptomatic or minimally evident for a long time. A careful clinical evaluation, reinforced by imaging findings, should help to distinguish asymptomatic disc derangements from pathologic conditions that may require treatment.

The first step of an MRI study of the TMJ is, without a doubt, to evaluate the disk, its morphology, and location in the closed- and open-mouth position with respect to the condyle. However, special attention must be paid to analyze the presence of other indirect and early signs that can result in the diagnosis of TMJ dysfunction. The last stage of dysfunction is announced on the appearance of osteoarthritic changes such flattening or condylar osteophytes. It is important for the radiologist to detect early MRI signs to thereby avoid the evolution to these advanced and irreversible phase of dysfunction with osteoarthritic changes.

\section{CONFLICT OF INTEREST}

The authors confirm that this article content has no conflict of interest.

\section{ACKNOWLEDGEMENTS}

Declared none.

\section{REFERENCES}

[1] Okeson JP. Functional anatomy and biomechanics of masticatory system. $7^{\text {th }}$ ed. Management of Temporomandibular Disorders and Occlusion, 2013; pp. 1-20.

[2] Kannan A, Sathasivasubramanian S. Comparative study of clinical and Magnetic resonance imaging diagnosis in patients with internal derangement of temporomandibular joint. J Indian Acad Oral Med Radiol 2011; 23: 569-75.

[3] Yildirim D, Dergin G, Tamam C, Moroglu S, Gurses B. Indirect measurement of Temporomandibular joint elasticity with Magnetic Resonance Imaging. Dentomaxillofac Radiol 2011; 40: 422-8.

[4] Wadhwa S, Kapila S. TMJ disorders: future innovations in diagnostics and therapeutics. J Dent Educ 2008; 72: 930-47.

[5] Maizlin ZV, Nutia N, Dent PB, et al. Displacement of temporomandibular joint disc: Correlation between clinical findings and MRI characteristics. J Can Dent Assoc 2010; 76: 1-5. 
[6] Modi P, Shaikh SS, Munday A. A cross sectional study of prevalence of temporomandibular joints in university students. Int J Sci Res Publ 2012; 2(9): 1-3.

[7] Ryalata S, Baqaina ZH, Amina WM, Sawaira F, Samarab O, Badranb DH. Prevalance of temporomandibular joint disorders among students of university of Jordan. J Clin Med Res 2009; 1(3): 15864.

[8] Mutlu N, Herken H, Guray E, Oz F. Evaluation of the prevalence of temporomandibular joint disorders syndrome in dental school students with Psychometric analysis. Turk J Med Sci 2002; 32: 345-50.

[9] Jerolimov V. Temporomandibular disorders and Orofacial pain. J Med Sci 2009; 33: 53-77.

[10] Ribeiro RF, Tallents RH, Katzberg RW, et al. The prevalence of Disc Displacement in symptomatic and asymptomatic volunteers aged 6 to 25 years. J Orofac Pain 1997; 11: 37-47.

[11] Campos MIG, Campos PSF, Cangussu MCT, Guimaraes RC, Lines SRP. Analysis of magnetic resonance imaging characterstics and pain in temporomandibular joint with and without degenerative changes of condyle. Int J Oral Maxillofac Surg 2008; 37: 529-34.

[12] Sener S, Akgunlu F. MRI characteristics of anterior disc displacement with and without reduction. Dentomaxillofac Radiol 2004; 33: 245-52.

[13] Gil C, Santos KCP, Dutra MEP, Kodaira SK, Oliviera JX. MRI analysis of the relationship between bone changes in the TMJ and articular disc position in symptomatic patients. Dentomaxillofac Radiol 2012; 41: 362-77.

[14] Larheim TA, Westesson PL, Sano T. Temporomandibular Joint Disc Displacement. Comparison in asymptomatic volunteers and patients. Radiology 2001; 218: 428-32.

[15] Herb k, Cho S, Stiles MA. Temporomandibular joint pain and dysfunction. Curr Pain Headache Rep 2006; 10; 408-14.

[16] Koh KJ, List T, Peterrson A, Rohlin M. Relationship between clinical and magnetic resonance imaging diagnosis and findings in degenerative and inflammatory Temporomandibular diseases: A systemic literature review. J Orofac Pain 2009; 23: 123-39.

[17] Manfredini D, Nardini LG, Winocur E. Research diagnostic criteria for temporomandibular disorders: a systemic review of axis 1 epidemiological findings. Oral Surg Oral Med Oral Path Oral Radiol Endod 2011; 112(4): 453-62.
[18] Clark GT, Delcanho RE, Goulet JP. The utility and validity of the current diagnostic procedures for defining Temporomandibular disorder patients. Adv Dent Res 1993; 7: 97-112.

[19] Benoliel R, Sharav Y. Masticatory Myofacial pain and tension type and chronic daily headache. J Orofac Pain Headache 2008:109-28.

[20] Koh KJ, List T, Peterson A, Rohlin M. Relationship between clinical and MRI diagnosis and findings of degenerative and inflammatory of Temporomandibular joint disease: A systemic literature review. J Orofac Pain 2009; 23:123-39.

[21] Milano V, Desiate A, Bellino R, Garofalo T. Magnetic Resonance Imaging of Temporomandibular disorders: classification, prevalence and interpretation of disc displacement and deformation. Dentomaxillofac Radiol 2000; 29: 352-61.

[22] Takatsuka S, Yoshida K, Ueki K, Marukawa K, Nakagawa K, Yamamoto E. Disc and condyle translation in patients with Temporomandibular disorder. Oral Surg Oral Med Oral Pathol Oral Radiol Endod 2005; 99: 614-21.

[23] Katzberg RW, Tallents RH. Normal and abnormal Temporomandibular joint disc and posterior attachment as depicted by Magnetic Resonance Imaging in symptomatic and asymptomatic subjects. J Oral Maxillofac Surg 2005; 63: 1155-61.

[24] Morrow D, Tallent RH, Katzberg RW, Murphy WC, Hart TC. Relationship of other joint problems and anterior disc position in Symptomatic TMD patients and asymptomatic volunteers. J Orofac Pain 1996; 10: 15-20.

[25] Maydane AV, Tesch RD, Denardin OVP, Dworkin SF. Possible etiologic factors in temporomandibular disorders of articular origin with implications for diagnosis and treatment. Dent J Orthop 2010; 15: 78-86.

[26] Benbelaid R, Fleiter B. Sensitivity and specificity of a new MRI method evaluating temporomandibula joint disc condyle relationship- in vivo study. Surg Radiol Anat 2006; 28: 71-5.

[27] Shaefer JR, Riley CJ, Caruso P, Keith D. Analysis of criteria for MRI diagnosis of TMJ Disc Displacement and Arthralgia. Int J Dent 2012; 2012: 1-8.

[28] Aoyama S, Kino K, Amagasa T, et al. Clinical and Magnetic resonance imaging studyof unilateral sideways discdisplacements of Temporomandibular joint. J Med Dent Sci 2002; 49: 89-94.

Received: June 20, 2014

Revised: November 17, 2014

Accepted: May 05, 2015

(C) Kumar et al.; Licensee Bentham Open.

This is an open access article licensed under the terms of the Creative Commons Attribution Non-Commercial License (http://creativecommons.org/licenses/by-nc/3.0/) which permits unrestricted, non-commercial use, distribution and reproduction in any medium, provided the work is properly cited. 